\title{
Online Collaborative Learning in Urology
}

\author{
Yi Li $^{1}$ (D) Nora G. Kern ${ }^{2} \cdot$ Simon L. Conti ${ }^{3} \cdot$ Lindsay A. Hampson ${ }^{1}$
}

Accepted: 24 October 2021 / Published online: 16 December 2021

(c) The Author(s), under exclusive licence to Springer Science+Business Media, LLC, part of Springer Nature 2021

\begin{abstract}
Reason for Review A recent shift towards use of telehealth and remote learning has significant implications on resident and fellow education in urology. Implementation of multi-institutional online didactic programs, spurred on by the COVID epidemic, has changed the traditional resident teaching paradigm from individual institutional silos of knowledge and expertise to a shared nationwide database of learning.

Recent Findings In this article, we explore the current trend towards virtual education and its progress to date, lessons learned on the optimization of this teaching modality, and future direction and sustainability of collaborated, standardized and accessible didactic education in urology.

Summary Multi-institutional collaborative remote video didactics has emerged as a critical part of resident education. These lectures have been overwhelmingly successful and have persisted beyond the pandemic to become a part of the urologic training curricula. This collaborative and standardized approach to resident education provides access to national and international experts, encourages cross-institutional collaboration and discussion, and builds a repository of lectures with easy access for learners. Utilization of this teaching modality will continue to be impactful in urologic training and will require ongoing efforts and input from both collaborating intuitions and professional societies to continue to improve on and engage in this important learning tool.
\end{abstract}

Keywords Resident education · Online didactics $\cdot$ Collaborative education

\section{Introduction}

Urological residency training programs provide clinical and surgical experience for trainees through the teaching hospital model, and in general provide didactic education through lectures given by local faculty and textbook-oriented selfdirected learning. This model for didactics relies on each specific institution creating its own curriculum and learning plan, resulting in significant heterogeneity among residency programs. Some institutions are limited by subspecialty

This article is part of the Topical Collection on Education

Yi Li

yi.li3@ucsf.edu

1 Department of Urology, University of California, San Francisco, San Francisco, CA, USA

2 Department of Urology, University of Virginia, Charlottesville, VA, USA

3 Department of Urology, Stanford University, Stanford, CA, USA topics without any expert input, and overall results in silos of knowledge and expertise [1]. This type of resident education has remained relatively stable for decades, with some advancements made in the last decade in terms of technique acquisition through hands-on skills labs.

A silver lining to the coronavirus pandemic was that it pushed programs to adopt a new way of educating trainees, given that in-person didactic teaching could not be carried out. With this change came the implementation of collaborative multi-institutional online didactic programs which brought with them new teaching systems that provide cross-institutional collaboration, shared and standardized expertise, and a repository of education materials for future learners [2].

In this article we explore (1) the trend towards virtual learning and what progress has been made, (2) lessons learned on how to best deliver this teaching most effectively, and (3) looking forward on how we might optimize and sustain this momentum towards a more collaborative, standardized, and accessible form of didactic education. 


\section{The Trend Towards Virtual Learning: A Pandemic Catalyst}

The 2019-2020 coronavirus pandemic had a major impact on all aspects of healthcare. During the initial shelter in place period, decreases in clinical volume, surgical volume, and the need to maintain social distancing resulted in significant changes to the residency experience in academic urology programs. This notable impact on residency training has been reported around the world during this period [3-8]. A survey study sent to 144 urology residency programs in the USA during the shelter in place period reported $92 \%$ of programs had a formal reduction of resident presence at work, $83 \%$ of programs had reduced overall resident work hours, and $60 \%$ of programs reported a concern that residents would not meet minimum case requirements [8]. Survey results from the Urology Collaborative Online Video Didactic (UrologyCOViD) lecture series post-lecture evaluations also reported that less than half of these programs saw any increase in didactics or lecture-based resident education during this time of decreased clinical education [9].

As a result of this decrease in clinical education opportunities, multiple collaborative webinar lecture series were formed during the height of the pandemic. The earliest of such programs was spearheaded by the urology department at the University of California, San Francisco in collaboration with seven other institutions. Within 1 week of its launch, the UrologyCOViD lecture series had filled 84 hourlong lecture slots from faculty volunteers from around the world. The first 2 weeks of lectures averaged over 400 live viewers on the Zoom platform and had over 7000 YouTube views of the lecture recordings [9].

Following this example, New York Section of AUA developed the Educational Multi-Institutional Program for Instructing Residents (EMPIRE) to similar success, and fellowship-specific lecture series soon followed (Table 1) [2]. The use of social media, a well-organized website, and free access to both live and recorded lectures on YouTube were critical components in the success of these programs.

Viewers of these lecture series were from all levels of training and across the globe, allowing for international discourse utilizing the chat and Q\&A functions of the webinars [9]. The impact of these cross-institutional programs and the familiarity with webinars and virtual meetings that developed during this period resulted in increased usage of these platforms in other academic settings. Virtual grand rounds broadcasts, panel discussions, and social media journal clubs have become commonplace, allowing for increased access and reach for these educational activities as well $[10,11]$.

\section{Lessons of Virtual Learning: How to Optimize Virtual Education}

With this shift towards virtual teaching and recorded lectures, it became important for programs and educators to better understand how to best utilize this virtual modality.

The European Society of Residents in Urology (ESRU) published a Twitter recruitment-based study that surveyed resident perspectives of virtual learning modalities and contents with over 500 resident responses recorded. The responders reported that the most useful virtual modality were pre-recorded videos, followed by interactive webinars, podcasts, and finally social media. The most useful content as reported by the survey were guidelines and surgical techniques [12].

Similarly, a review of over 2000 post-lecture evaluations in the UrologyCOViD lecture series was performed to compare the best received lecture style within the UrologyCOViD lectures. Eighty-four lectures given between March and May of 2020 were reviewed by senior residents at UCSF and categorized as guideline-based, case-based, practice updates, or surgical technique based. Viewer feedback on a Likert scale regarding instructor knowledgeability, lecturer effectiveness, subject area relevance, and usefulness to learning were analyzed, and multivariate analysis was performed controlling for lecturer career stage, lecture topic, and viewer training level. In this study, case-based and guideline-based lectures were the best received lectures by viewers [13].

The actual impact of these lecture styles may be dependent on the training level of the learner. Guideline-based lectures are certainly useful and relevant to junior learners. Practice update lectures assume some knowledge of the topic and are more relevant for senior learners. Case-based lectures may mimic the "flipped classroom" approach and can engage learners with interaction, and surgical technique
Table 1 Urology virtual education series

\begin{tabular}{lll}
\hline Institution & Series & Acronym \\
\hline UCSF & Collaborative Online Video Didactics Lecture Series & COViD \\
NY Section AUA & Educational Multi-institutional Program for Instructing REsidents & EMPIRE \\
UCSF & Pediatric Urology Fellowship Lectures Online & PedsUroFLO \\
EDGE Consortium & Endourology Disease Group for Excellence Talks & EDGE Talks \\
SSMR/SMRU & SSMR Male Infertility Lecture Education Series & SMILES \\
Case Western & Genitourinary Reconstruction Online Learning Series & N/A \\
\hline
\end{tabular}


lectures may be more effective when focused on less commonly seen surgical techniques, making them more relevant to advanced learners.

A recent unpublished trainee conjoint analysis survey on preferences for didactic education where trainees were asked to select their preferred method of delivery, presentation style, presenter credentials, and curriculum design also demonstrated learner preferences. Medical students, residents, and fellows all preferred online over in-person didactic sessions, leading experts from various institutions over their own local faculty, and standardized shared nationwide curriculum over individual institution designed teaching. The more senior trainees showed a stronger preference for online/à la carte lectures, likely due to their familiarity with the content and ability to pick and choose [14].

These studies evaluating trainee feedback are the first steps to better understand how to optimize virtual learning. It is clear that trainees value virtual learning; interactive webinars and pre-recorded videos both provide easy access and the ability to reach a much wider audience than the traditional model. To truly evaluate effectiveness of teaching, studies are required that incorporate pre- and post-lecture knowledge retention evaluation. In one recent unpublished study evaluating the effectiveness of the UrologyCOViD lecture series, residents who watched 3-5 out of 5 lectures scored higher on a knowledge-based test compared to those who watched less. Additionally, although residents had increasing test scores by post-graduate year prior to watching the lecture series, there was no significant difference in test scores after watching the series by post-graduate year. This may indicate the benefit these types of lectures have on the more junior learners [15].

\section{Future of Virtual Learning: Where We Go from Here}

The initial feedback regarding the collaborative multiinstitutional programs were overwhelmingly positive. For both the COViD and EMPIRE series, feedback from viewers reported over $90 \%$ positive impressions and nearly $100 \%$ of viewers desiring that the lecture series continue into the future $[2,9]$.

With the resumption of more regular clinical schedules, the collaborative video didactic series borne out of the educational vacuum during the pandemic evolved. The UrologyCOViD series transitioned to once weekly lectures and has also become available in podcast form. The webinars are now pre-recorded and continue to be actively viewed on all platforms. The NYAUA EMPIRE lecture series has similarly adapted to the resumption of clinical activities. The series broadcasted an in-service review series prior to the resident in-service exam, and is currently providing "Hidden Curriculum" lecture series which has been highly attended.

Video-based learning has been shown to be effective in resident education [16]. YouTube channels with recorded lectures continue to be highly accessed, and pre-recorded lectures have been incorporated in various teaching curriculums at local programs as well as virtual urology subinternships. UrologyCOViD and EMPIRE YouTube videos are highly utilized study resources for exams and content in general. It would appear that the virtual education aspect of resident learning will be here to stay for the time being.

Unsurprisingly, there have been calls for a national video based-curriculum for resident learning [17]. Tabakin et al. argues that professional urologic societies such as the AUA and the Society of Academic Urology should organize committees to curate video lectures of guidelines, surgical techniques, and practice updates. Such a revamping could be an upgrade of the current AUA Core Curriculum, and utilize the lessons and formats developed by these independent lecture series, while also incorporating other clinical training scenarios such as with surgical simulators and patient cases. While the utilization of remote video-based learning in urologic training is not a new idea [18], the timing may be ripe now for societies to take advantage of the momentum for collaborative online teaching to further standardize and improve resident education.

The benefits of virtual education extend beyond the benefits for individual leaners. Virtual education allows for more widespread standardization of topics and training and can help to bridge gaps in individual programs that may not have experts in all areas. Additionally, it can further push academic institutions to utilize our new familiarity with webinars and online meetings to continue to collaborate in other areas. Zoom journal clubs where the authors are invited to participate in the discussions, or where multiple residency programs have joined together to discuss latest impact articles have become normalized. The technology and culture for improving and standardizing didactic learning are now available, and it will be up to both the academic institutions and the learners to take advantage of what it offers.

\section{Conclusion}

Multi-institutional collaborative remote video didactics has emerged as a critical part of resident education, spurred on by the COVID-19 pandemic. These lectures have been overwhelmingly successful and have persisted beyond the pandemic to become a part of the urologic training curricula. This collaborative and standardized approach to resident education provides access to national and international experts, encourages cross-institutional collaboration 
and discussion, and builds a repository of lectures with easy access for learners. Utilization of this teaching modality will continue to be impactful in urologic training and will require ongoing efforts and input from both collaborating institutions and professional societies to continue to improve on and engage in this important learning tool.

\section{Compliance with Ethical Standards}

Conflict of Interest The authors declare no competing interests.

Human and Animal Rights and Informed Consent This article does not contain any studies with human or animal subjects performed by any of the authors.

\section{References}

1. Mann U, Ramjiawan R, Nayak JG, et al. Heterogeneity in urology teaching curricula among Canadian urology residency programs. Can Urol Assoc J. 2020;15.

2. Smigelski M, Movassaghi M, Small A. Urology virtual education programs during the COVID-19 pandemic. Curr Urol Rep. 2020;21.

3. Khusid JA, Weinstein CS, Becerra AZ, et al. Well-being and education of urology residents during the COVID-19 pandemic: results of an American National Survey. Int J Clin Pract. 2020;74.

4. Prezotti JA, Henriques JVT, Favorito LA, et al. Impact of COVID19 on education, health and lifestyle behaviour of Brazilian urology residents. Int Braz J Urol. 2021;47:753-76.

5. Teixeira BL, Cabral J, Mendes G, et al. How the COVID-19 pandemic changed urology residency - a nationwide survey from the Portuguese resident's perspective. Cent Eur J Urol. 2021;74:121-7.

6. Yee C, Wong H, Tam MH, et al. Effect of SARS and COVID-19 outbreaks on urology practice and training. Hong Kong Med. J. 2021.
7. Amparore D, Claps F, Cacciamani GE, et al. Impact of the COVID-19 pandemic on urology residency training in Italy. Minerva Urol e Nefrol. 2020;72:505-9.

8. Rosen GH, Murray KS, Greene KL, et al. Effect of COVID19 on urology residency training: a nationwide survey of program directors by the Society of Academic Urologists. J Urol. 2020;204:1039-45.

9. Li Y, Chu C, de la Calle CM, et al. Multi-institutional collaborative resident education in the era of COVID-19. Urol Pract. 2020.

10. Manning E, Calaway A, Dubin JM, et al. Growth of the twitter presence of academic urology training programs and its catalysis by the COVID-19 pandemic. Eur Urol. 2021.

11. Bayne CE, Cardona-Grau D, Hsieh MH. Introducing the Pediatric Urology Journal Club on Twitter. J Pediatr Urol. 2017;13:2-3.

12. Campi R, Amparore D, Checcucci E, et al. Exploring the residents' perspective on smart learning modalities and contents for virtual urology education: lesson learned during the COVID-19 pandemic. Actas Urol Esp. 2021;45:39-48.

13. Li Y, de la Calle CM, Chu C, et al. Case-based and guidelines based lecturers are the most preferred form of online resident education: results from the Urology Online Video Didactic series (COViD). Urology. 2021.

14. Spradling K, Seufert C, Conti SL. Evaluation of urology trainee preferences in didactics education: an international choice-based conjoint analysis.

15. Tuong M, Winkleman A, Yang J, et al. Evaluation of the educational impact of the Urology Collaborative Online Video Didactics Lecture Series.

16. Green JL, Suresh V, Bittar P, et al. The utilization of video technology in surgical education: a systematic review. J Surg Res. 2019;235:171-80.

17. Tabakin AL, Patel HV, Singer EA. Lessons learned from the COVID-19 pandemic: a call for a national video-based curriculum for urology residents. J Surg Educ. 2021;78:324-6.

18. Cook A, Salle JLP, Reid J, et al. Prospective evaluation of remote, interactive videoconferencing to enhance urology resident education: the genitourinary teleteaching initiative. J Urol. 2005;174:1958-60.

Publisher's Note Springer Nature remains neutral with regard to jurisdictional claims in published maps and institutional affiliations. 